\title{
Structure of Particle Assembly in Water-Based Magnetite Magnetic Fluid Under Zero Applied Field
}

\author{
B. Jeyadevan*, I. Nakatani**, H. Oka***, and K. Tohji* \\ ${ }^{*}$ Tohoku University, Aramaki aza Aoba 01, Sendai 980-8579, Japan \\ ${ }^{* *}$ National Research Institute for Metals, 1-2-1 Sengen, Tsukuba City 305-0047 \\ **** Iwate University, Morioka City 020-0066
}

Received on 15 February, 2001

\begin{abstract}
The magnetic particle dispersion in magnetic fluid has been found to depend very much on the type of dispersing media. The water-based magnetic fluid has been found to contain large number of inherent clusters even in zero applied magnetic field. In this paper, we discuss the possible origin of these primary clusters and propose that these clusters are of magnetic origin. The scanning probe microscope observation of primary clusters were carried out and found to be of ring shape.
\end{abstract}

\section{Introduction}

Magnetic fluids are systems consisting of colloidal suspension of near-spherical magnetic particles in polar and non-polar solvents. Depending on the type of solvent, the particles are either coated with single or double layers of surfactant to prevent coalescing at room temperature. Among water and hydrocarbonbased magnetic fluids, long-term stability of the waterbased magnetic fluid has been found to be comparatively poor. Nakatani reported the presence of large number of inherent clusters of about $200 \mathrm{~nm}$ in diameter in water-based magnetic fluid even under zero applied magnetic field [1]. However, ionic magnetic fluid that also uses water as solvent [2] and kerosene-based magnetic fluid did not show any inherent clusters. The reasons for these differences in microscopic level among different types of magnetic fluids are unknown. Therefore, identifying the particle structures under zero and applied magnetic field as well as their influence on the physical properties are considered important. In this paper, we have focused only on water-based magnetic fluid and report our observations on particle structures under zero applied field in micro and nanometer levels. And also, discuss the possible reasons for the formation of these particle structures.

\section{Experimental}

The water-based magnetic fluid (W40) used in this study is a product of Taiho Co. Ltd. The micrometer scale observations were made on a thin liquid film of magnetic fluid in an ultra microscope setup described in ref. [2]. Nano-scale images of the magnetic particles in magnetic fluid were obtained by using Scanning Probe Microscope (SPM-9500J, Shimadzu, Japan).

\section{Results and Discussion}

In the ultramicroscope, the presence of any object is visualized due to Rayleigh scattering of light by the object and the detectable limit depends on the scattering intensity of the object. Here, the light scattering intensity mainly depends on particle diameter. As reported earlier[1], in the water-based MF, we observed extremely large number of agglomerated magnetic particles (primary clusters) that scatter specks of light even under zero applied field as shown in Fig. 1. The scattered light intensity show a variation with time suggesting rotation of the non- spherical object. However, the resolution of the ultramicroscope does not provide any further information about the shape of inherent clusters.

On the other hand, Jund et al. [3] discussed the stability and fragmentation of complex structures in magnetic fluid based on quaternion molecular dynamics and suggested that rings are more stable than chains when the energy gain from connecting the chain ends outweighs the energy loss due to the misalignment of neighboring dipoles. For $\mathrm{N}$-membered chain and ring in zero field, their model gives the energy per particle as 


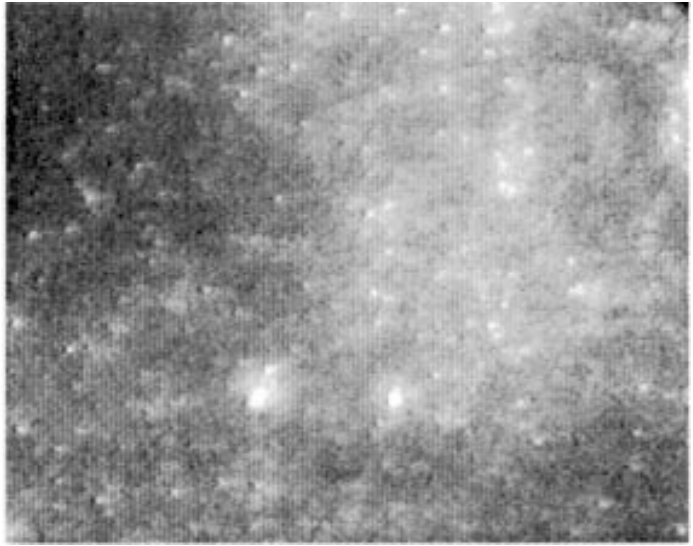

Figure 1. Ultra micrographs of water-based magnetic fluid under zero applied fields.

$$
\begin{gathered}
E_{\text {chain }}^{0}(N) / N \bullet-\alpha\left(2 \mu_{0}^{2} / \sigma^{3}\right)(1-1 / N) . . \\
E_{\text {ring }}^{0}(N) / N \bullet-\alpha\left(2 \mu_{0}^{2} / \sigma^{3}\right)[3+\cos (2 \pi / N)] / 4 \ldots
\end{gathered}
$$

$E_{0}$ - energy, $\alpha$ - constant, $\sigma$ - particle diameter, $\mu_{0}$ magnetic moment.

Energy per particle in a chain or ring depends on the number of particles that forms the structure. In zero field rings are favored over chains for all systems more than three particles.

Considering the reason for the presence of large number of inherent clusters in zero applied field in water-based magnetic fluid compared to hydrocarbonbased or ionic magnetic fluid, it can be either due to the difference in the preparation technique or the presence of considerable fraction of comparatively larger particles in the suspension that induces stronger magnetic interaction.

Considering the reason for the presence of large number of inherent clusters in zero applied field in water-based magnetic fluid compared to hydrocarbonbased or ionic magnetic fluid, it can be either due to the difference in the preparation technique or the presence of considerable fraction of comparatively larger particles in the suspension that induces stronger magnetic interaction.

During synthesis of water-based magnetic fluid, the magnetite particles are initially adsorbed with oleate where, hydrophilic end of oleate chemisorbs on the particle surface and the hydrophobic-end face the solvent water. Thus, the particles do not acclimatize themselves with surrounding and begin to coagulate. Thereafter, the SDBS that physisorbs as the second layer is added only to these aggregates. Though some of them disperse in water as individual particles, large percent of them remain as aggregates. In this case, the aggregates formed will not be of any particular size or shape. On the other hand, inherent clusters can also be induced by the presence of large fraction of non-super paramagnetic particles. The potential energy calculations suggests that the diameter of particle stably dispersed in a solvent depends also on the thickness of the surfactant layer; thicker the surfactant layer, larger the particle diameter dispersed stably[4]. Since the magnetic particle dispersed in polar solvent have thicker surfactant layer than the ones dispersed in non-polar solvents, it is reasonable to assume that all other factors being equal, the maximum diameter of the particles dispersed in waterbased magnetic fluid to be higher than kerosene-based magnetic fluid. Furthermore, at any specific temperature, the magnetic behavior of these particles is size dependent. It has been observed that even kerosene based magnetic fluid consists considerable fraction of non-superparamagnetic particles at room temperature [5], thus, water-based magnetic fluid is expected to have more due to the ability to disperse particles of larger diameter. Therefore, when there is a strong dipole-dipole interaction between particles in the system, formation of either chain or ring in zero applied can be expected.
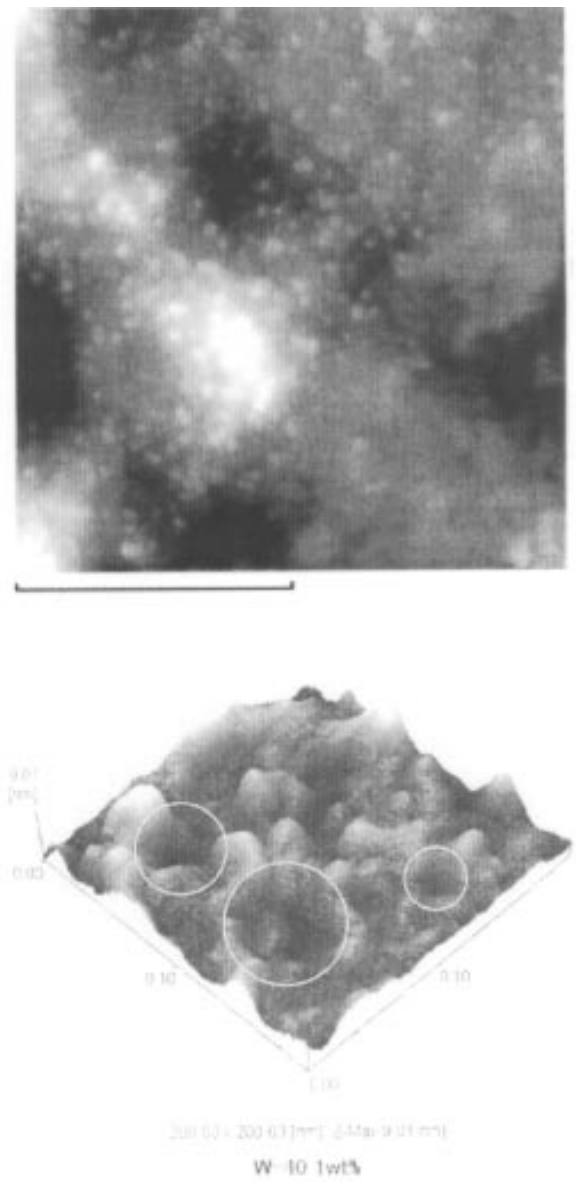

Figure 2. Scanning Probe Micrographs of water-based magnetic fluid (a) scan area $1.00 \times 1.00 \mathrm{~nm}$ and (b) $200 \times 200 \mathrm{~nm}$.

To investigate the morphology of these particle structures under zero applied field, we made Scanning Probe Microscope (SPM) measurements on $1 \%$ waterbased magnetic fluid(W40). Since the measurement of particle structures in liquid is not possible, measurements were carried out on a dried drop of magnetic fluid. The drop of the water-based magnetic fluid was 
placed on a glass substrate and allowed to dry naturally. The SPM images of the dried sample is shown in Figs. 2 (a) and (b). In Fig. 2(a), we could observe accumulation of particle aggregates. However, when the area was magnified further (twenty five times) and the height of the surface of these aggregates were traced, we obseved some defined particle structures as shown in Fig. 2(b). We could clearly see some ring-shaped structures supporting the quaternion molecular dynamics simulations by Jund et al.. The number of particles forming the structure differ from ring to ring. We also could observe that the diameter of the particle participating in the structure are larger than $10 \mathrm{~nm}$ in diameter. Therefore, we could conclude that the presence of inherent clusters in water-based magnetic fluid are of magnetic origin and induced mainly by the presence of particles of larger diameter.

\section{Conclusion}

The inherent particle clusters observed in water-based magnetic fluid under zero applied fields were found to be of ring structures. The formation of the above struc- tures were believed to be due particle interaction initiated by larger particles dispersed in the system.

\section{Acknowledgement}

One of the author (B.J) wishes to thank Mr. B. Matsuda of Shimadzu Analytical and Measuring Center Inc., for the Scanning Probe Microscopy measurements.

\section{References}

[1] I. Nakatani, Proc. of the Intl. Symp. on Hydrodynamics of magnetic fluids and It's Application(ISHMFA '97) July 18-20th, p9 (1997).

[2] B. Jeyadevan and I. Nakatani, J. Magn. Magn. Mat. 201, 62 (1999).

[3] P. Jund, S. G. Kim, D. Tomanek and J. Hetherington, Phys. Rev. Lett. 74(15), 3049 (1995).

[4] R. E. Rosensweig, Ferrohydrodynamics, Cambridge, London (1985).

[5] B. Jeyadevan and K. Nakatsuka, Proc. of the Intl. Conf. on Ferrites (ICF 6), Tokyo and Kyoto, Japan, p.1713 (1992). 